\title{
LITERATURE HELPS WORLDING THE WORLD - A CONVERSATION WITH BERTRAND WESTPHAL
}

An interview with Bertrand Westphal, Professor of Comparative Literature at the University of Limoges, by Marius Conkan and Emanuel Modoc

Text in French

Recommended citation: Conkan, Marius, Modoc, Emanuel. "Literature Helps Worlding the World - A Conversation with Bertrand Westphal". Metacritic Journal for $\begin{array}{lllll}\text { Comparative Studies } & \text { and } & \text { Theory }\end{array}$ https://doi.org/10.24193/mjcst.2020.9.02

\begin{abstract}
In the following interview, Bertrand Westphal, professor of comparative literature at the University of Limoges, discusses some of the prevailing issues surrounding contemporary forays into spatial studies and the function of the humanities in current academia. The dialogue also touches on subjects pertaining to World Literature studies, such as Immanuel Wallerstein's "world systems analysis" or Franco Moretti's "distant reading" method, in an attempt to propose an applied and pragmatic approach through which geocriticism can become essential to the study of literature. Furthermore, Westphal engages in a highly stimulating examination of common spatial and cultural stereotypes such as Central and Eastern Europe, while also commenting on subjects like the interdependence of time and space in our understanding of cultural phenomena or geocriticism's applicability to both mimetic and abstract spaces.
\end{abstract}

Keywords: geocriticism, spatial turn, worlding, world systems analysis, distant reading

Interviewer: Dans la dernière partie de votre livre La Géocritique. Réel, Fiction, Espace, vous écrivez: "L'un des autres enjeux de la géocritique consiste non plus à dégager la corrélation entre le réel et la fiction, entre le monde et la bibliothèque, ou de la considérer comme une métaphore, mais de proposer une véritable hypothèse de travail. [...] Les études littéraires seront-elles 'applicables' en dehors de la bibliothèque ou des territoires de la fiction? En d'autres termes, l'étude de la littérature 
pourrait-elle nous aider à déchiffrer le monde ? Je le crois bien ». Compte tenu de la diminution de l'autorité des humanités, comment envisagez-vous donc l'emploi empirique de la géocritique en dehors de l'espace abstrait de la bibliothèque ?

Bertrand Westphal: Au moment d'écrire ces lignes, nous étions en 2005. J'avais la ferme conviction que la littérature et les mondes fictionnels avaient un véritable rôle à jouer au niveau du référent, dans la mesure où les rapports entre référent et représentation n'étaient pas destinés à demeurer unilatéraux mais qu'ils entraient dans une sorte de dialectique, quand bien même la relation n'est pas et n'a jamais été équilibrée. Le réel prime; il le fait d'autant plus que la sortie de l'ère postmoderne, une sorte de parenthèse enchantée, paraît désormais inéluctable. Je crois cependant qu'il convient de s'abstenir de dresser un panorama trop sombre de la situation dans laquelle se trouve la littérature et qu'il faut éviter de sous-estimer les effets concrets qu'elle peut produire. L'autorité des humanités diminue-t-elle vraiment? Oui, sans doute, mais pas au point de perdre tout prolongement dans nos sociétés. La littérature est - comme je le répète depuis longtemps, après Paul Ricœur - un «laboratoire des possibles ». En d'autres termes, elle expérimente et met en œuvre (au sens fort) toutes les ontologies imaginables et concevables dans un monde où les facultés d'expression paraissent de plus en plus limitées en dépit ou à cause du brouhaha dans lequel nous placent des réseaux sociaux et des médias omniprésents, manifestement inscrits dans la pure synchronie du streaming et du spectacle, dans la réaction épidermique. Cela m’inciterait à penser qu'à défaut de mieux le livre nous permet encore de cultiver une forme de longue durée, celle que nécessite la lecture d'un roman ! Un exercice de yoga, en quelque sorte ! Je plaisante, bien entendu, mais à peine.

En lisant Immanuel Wallerstein, qui nous a quittés à l'été 2019 et qui était un sociologue et un économiste, l'homme des world systems, je me suis aperçu qu'on pouvait même concevoir une démonstration par l'absurde. En effet, les humanités - et la littérature en particulier - sont d'autant plus importantes que tout pouvoir hégémonique désireux d'occuper le centre, où qu'il se trouve, s'efforce toujours d'asseoir sa domination sur le monde de la représentation et donc sur l'univers si particulier de la représentation littéraire. Si le pouvoir en place éprouve un tel sens de l'urgence, c'est que l'impact de la culture et de la littérature est plus important que ne le pensent... les 
littéraires. Peut-être acceptons-nous trop vite d'être rétrogradés à une fonction subalterne au sein de la société, comme si nous étions conditionnés depuis trop longtemps par des paramètres négatifs.

En tout cas, pour répondre précisément à votre question sur l'utilisation de la géocritique dans un environnement hors littérature, hors bibliothèque, j'avais très vite envisagé une relation possible entre littérature et " société civile » : celle, par exemple, qui prenait en considération le tourisme. Il n'était pas exclusivement question d'alimenter une critique, mais aussi de « faire du constructif » - par exemple agir sur le tourisme culturel et littéraire. De ce point de vue, la géocritique pouvait apporter un soutien méthodologique. Depuis quelques années, j'en suis revenu à des considérations plus théoriques, mais j'ai le sentiment qu'elles aussi sont connectées à un monde qui ne se réduit pas qu'à la bibliothèque. En anglais, on parle de worlding - un terme et un concept intéressants que la world literature (je songe en particulier à Pheng Cheah) a hérités de Heidegger tout en les transformant considérablement. Literature helps worlding the world, et peut-être que la géocritique aide d'autres théories à étudier l'impact de ce world worlding.

Interviewer: Vous définissez la géocritique comme une approche géocentrique à plusieurs couches des espaces réels et imaginaires, dont la description n'est pas limitée à la littérature. Dans son propre ouvrage, Spatiality, Robert T. Tally Jr, le traducteur du livre susmentionné, se pose la question suivante concernant votre méthode géocritique : "Qui décide quels seront les textes à inclure dans un corpus littéraire qui sera utilisé pour 'lire' un lieu déterminé ? [...] l'approche géocentrique de Westphal n'a pas réussi à éviter entièrement le problème du sujet». Dans ce contexte, quelle est votre position vis-à-vis de la subjectivité et de son rôle dans une lecture multifocalisée des espaces? Comment la géocritique pourrait-elle éviter d'être justement un simple processus de close-reading des espaces, fussent-ils réels ou fictionnelles?

Bertrand Westphal: La remarque de Robert Tally est tout à fait pertinente. Elle concerne le corpus sur lequel appuyer une analyse et pointe une aporie. En somme, que faire : opérer une sélection ou considérer que le corpus est illimité et que le choix est subjectif ? À mon avis, la solution ne consiste pas nécessairement à privilégier l'étude 
monographique pour éviter d'avoir à l'affronter - et, au demeurant, qu'est-ce qui guide la sélection monographique sinon un autre choix subjectif? Donner dans la monographie représenterait de surcroît un pis-aller peu acceptable pour un comparatiste, et laissons de côté la question de la définition de ce qu'est un ou une comparatiste aujourd'hui ! Au moment d'écrire La Géocritique, j'avais émis l'hypothèse que dans le cadre d'une approche géocentrée il convenait de dégager un corpus suffisant, à défaut d'être satisfaisant. Il s'agissait, a minima, de mettre en relation des points de vue différents articulés autour d'un même lieu. Je parlais alors de « seuil de représentativité». Selon une logique arithmétique, on pourrait être tenté de le quantifier. C'est évidemment impossible. J'avais d'ailleurs remarqué que si l'on trouve trois textes littéraires qui évoquent chacun une certaine tour d'une certaine bourgade sarde (Santa Lucia di Siniscola) le résultat était impressionnant. En revanche, si l'on pense pouvoir dire beaucoup de New York, de Bucarest ou de Buenos Aires à travers trois autres textes, dans une optique géocentrée, le résultat sera forcément décevant car il sera dérisoire. Faire une géocritique des métropoles n'aurait qu'un sens relatif; il faudrait à tout le moins spécialiser le focus. Là encore, tenter de quantifier n'aurait que peu de sens, voire aucun. La part du subjectif est bien sûr importante ; elle est inhérente aux sciences humaines, à toutes les sciences, et il faut savoir accepter sinon le fait accompli du moins le constat. La subjectivité peut être encadrée par la sélection de critères. J'avais évoqué une multifocalisation et l'émergence de trois catégories de points de vue majeures (endo-, -allo- et exogène), qui mériteraient sans l'ombre d'un doute d'être peaufinées car elles me paraissent un peu cloisonnées aujourd'hui. Par ailleurs, dans le domaine littéraire, ces modalités n'ont plus le même sens maintenant que la société se planétarise qu'au XIXe siècle, par exemple. Alors, en littérature, le point de vue exogène était surtout celui du voyageur, dans une acception conventionnelle $d u$ terme, relayée par l'imagologie, en littérature comparée ; aujourd'hui, le voyage a pris un sens infiniment plus complexe; ses déclinaisons sont quasiment innombrables dès lors qu'il inclut une dimension diasporique subie. Cela me fait préciser que dans un domaine géocritique l'étude ne saurait scinder espace et temps, sauf à conforter à son tour les stéréotypes qu'elle essaie de dénoncer.

Nous sommes en 2020 et mon opinion sur la question du corpus a évolué. Plus le temps passe, plus je me dis qu'elle est importante, pour ne pas dire déterminante. Ce 
constat s'appuie aussi sur les problèmes que rencontre la world literature, qui en tant qu'objet d'étude m’intéresse énormément - la géocritique me paraît particulièrement adaptée pour en examiner les principes -au moment de définir le canon qu'elle promeut et les corpus sur lesquels elle s'appuie. Prétendument objective dans ses stratégies de distant reading, elle me semble en réalité très subjective - même si cette subjectivité se développe à la très grande échelle de la langue, notamment, et du marché global de la traduction. Dans mes derniers essais (La cage des méridiens, 2016; L'atlas des égarements, 2019), qui ne sont pas traduits en anglais (ce dont je ne me plains pas, d'ailleurs, car si je le faisais je serais en contradiction avec mes propres écrits portant sur la globalisation), je me livre à une réflexion soutenue sur la cartographie, qui intègre également le domaine des arts visuels contemporains, si révélateur, si stimulant. Ce faisant, je me suis rendu compte, encore plus nettement que par le passé, que la dichotomie ne concerne pas ce qui est censé être objectif (la pseudo-scientificité) et subjectif, mais plutôt, d'une part, une attitude ethnocentrique implicite ou tue, ouverte sur l'erreur de parallaxe et de facto entérinée et, d'autre part, une posture ethnocentrique mise en cause - ce qui a le mérite d'être honnête, je crois - et aussitôt corrigée, au maximum du possible, par un effort de défamiliarisation, comme diraient Paul Gilroy ou Rosi Braidotti, et par la relativisation de prédicats qui ne seront pas considérés comme des affirmations définitives et des manifestations aiguës de pensée forte. Ma formation à la fois postmoderne et postcoloniale continue de toute façon à me rendre méfiant à l'égard des déclarations trop assertives, héritières d'une arrogance universaliste. Celle-ci n'est d'ailleurs pas un monopole eurocentrique, si, par « eurocentrique », on entend « relatif à la seule Europe » (je songe à la production étasunienne, en particulier).

Interviewer: En complément de la question précédente : dans quelle mesure votre approche géocritique pourrait-elle s'intégrer à la notion de «distant-reading » et à la méthode quantitative proposes par Franco Moretti?

Bertrand Westphal: Franco Moretti a écrit des essais qui m’ont énormément inspiré, notamment Atlas of the European Novel, qui a paru en italien en 1997, avant d'être traduit en anglais l'année suivante, chez Verso. Qu'il le veuille ou non, Moretti s'inscrit 
dans le sillage d'une école italienne dont on parle certes, mais qui mériterait de figurer davantage au premier plan. Je me réfère surtout à Claudio Magris, dont Danube, paru en 1986, a été une véritable révélation pour moi et pour tant d'autres. L'art de dire des choses érudites tout en développant un art consommé de l'écriture! Umberto Eco a illustré cela, tout comme Massimo Cacciari. Ce dernier a emprunté quelques hypothèses à la géophilosophie deleuzienne pour les interpréter à sa manière, passionnante. Ce ne sont pas les seuls grands noms, d'ailleurs. J'ai été frappé par la manière dont Moretti dégageait une cartographie, au sens géographique du terme, dans des œuvres qui n'avaient pas vocation, a priori, à une lecture de ce genre. Il a été parmi les premiers à le faire et il a constitué une source d'inspiration pour beaucoup de théoriciens et de théoriciennes de la littérature. Les principes du distant reading sont fondamentaux pour comprendre la circulation des œuvres au niveau global. Moretti a disposé de moyens considérables à Columbia puis à Stanford; il a su les utiliser au mieux pour analyser des corpus monumentaux auxquels une seule et même personne n'aurait aucune chance d'avoir accès. Nous sommes confrontés en permanence à l'infini culturel et de plus en plus à l'heure où la culture se planétarise ou emprunte le chemin d'un Planetarian Turn, comme dit si bien Christian Moraru. J'ai eu l'occasion de lire Distant Reading à sa sortie aux Etats-Unis, en 2013, alors que je me trouvais pour deux ans à UNC Charlotte, où j'ai écrit La cage des méridiens. Le texte de Moretti est abondamment cité dans cet essai. En ce moment, reclus à domicile comme une partie des habitants et des habitantes de la planète, je suis justement en train d'écrire un nouveau livre sur l'infini culturel. Il va de soi qu'un des chapitres porte sur l'examen de méthodes quantitatives, car, soit dit en passant, je suis conscient d'avoir trop longtemps négligé la sociologie de la littérature pour aborder les questions du global. Le marché, la traduction, l'édition... autant de terrains d'études où les indicateurs quantitatifs nous permettent de proposer quelques hypothèses. C'est ce qu'a fait Moretti ; c'est aussi ce que fait quelqu'un comme Gisèle Sapiro en France.

Interviewer: Dans Geocritical Explorations: Space, Place, and Mapping in Literary and Cultural Studies (édité par Robert T. Tally Jr.) vous montrez que «la géocritique ne privilégie plus un point de vue unique, mais embrasse une vision éclectique de la notion d'espace. [...] cette hybridation des différents objectifs (focalisation multiple) 
tend à relativiser l'altérité et à identifier les stéréotypes ». La géocritique peut-elle identifier et aussi éviter les stéréotypes dans son décodage de l'espace de l'Europe centrale et orientale, en traversant toutes les étapes de sa représentation historique, depuis son orientalisation initiale, en passant par l'étape de la Mitteleuropa pendant la domination impériale et de sa description, dans l'entre-deux-guerres, comme un "Autre proche » et jusqu’à sa régression présumée pendant le régime communiste?

Bertrand Westphal: Voilà une question fort précise qui s'articule autour de plusieurs points. Pour ce qui est de savoir si la géocritique est en mesure d'éviter les stéréotypes quand elle véhicule l'examen des espaces d'Europe centrale et orientale, je répondrais : et pourquoi pas? Mais j'aurais un peu de mal à affirmer quoi que ce soit, vu que je risquerais de me retrouver dans la position de l'avocat de la défense ! En tout cas, je me rends compte que beaucoup de colloques ont été consacrés à cette approche dans les parties de l'Europe concernées, ces dernières années (Macédoine, Pologne, Slovénie etc.). Je m'en réjouis mais n'en suis pas surpris. J'ai moi-même eu l'occasion de coorganiser un colloque à Cluj-Napoca avec Corina Moldovan voici un certain nombre d'années; il portait sur une «géocritique de la Transylvanie » et avait rassemblé de nombreux chercheurs et chercheuses internationaux. De manière plus générale, j'aurais tendance à dire que du fait de l'extraordinaire complexité des stratigraphies spatiotemporelles caractérisant l'Europe centrale et orientale, la géocritique devrait pouvoir apporter quelques instruments utiles, du fait que justement elle conjugue espace et temps en vouant une attention particulière à la profondeur diachronique, qu'elle prête attention à la multiplicité des points de vue et qu'elle interroge le statut de celui ou celle qui entreprend l'examen des lieux.

Bien entendu, je ne puis que répéter ce que je remarquais tout à l'heure : il est impossible de prendre en compte l'ensemble de l'aire, que ce soit dans la durée ou dans l'instant. J'ai abordé plusieurs fois le sujet au fil des années, car il me passionnait, sans parler cependant aucune des langues pratiquées dans cette grande région du monde, à l'exception de l'allemand qui, le long du Danube, n'est pas une langue marginale. Influencé par Magris, j'ai parlé de la Mitteleuropa, surtout sur son versant autrichien, via des œuvres de Milo Dor ou d'Elias Canetti, notamment. Je me suis aussi brièvement concentré sur la Bulgarie, à un autre moment, plus lointain, et sur l'Albanie et la côte 
adriatique de l'ancienne Yougoslavie (dans L'œil de la Méditerranée, 2005). Plus récemment, toujours à Charlotte, $\mathrm{NC}$, j'ai co-dirigé avec Lauren Lydic un volume sur les lendemains des guerres yougoslaves (Le silence et la parole au lendemain des guerres yougoslaves, 2015) et repris en 2019, sous un angle différent (quantitatif !), une étude géocritique d'Istanbul. Il serait totalement absurde de vouloir examiner l'Europe centrale et orientale dans un même mouvement, surtout si on s'efforçait de le faire en diachronie. Là, vraiment, sauf à se spécialiser sur un thème très précis, on aboutirait presque à coup sûr au stéréotype, me semble-t-il. A vrai dire, plus on pousse l'étude, plus on se rend compte combien les clichés constituent une simplification abusive face à un ensemble - pour peu que l'on puisse parler d' 'ensemble' - totalement hétérogène. La Mitteleuropa de Claudio Magris est fascinante, mais, en définitive, existe-t-elle ? Correspond-elle à l'Europe médiane, qui serait sa traduction en français ? Non, car l'Europe médiane aurait elle-même une autre définition, plus proche de celle que lui a conférée Czesław Miłosz! Et quid de l'Europe centrale? Quid aussi de l' « Europe de l'Est », qui, jadis opposée à l' " Europe de l'Ouest », semble s'être estompée au profit de l'« Europe centrale », après la chute du Mur ? La terminologie est riche en connotations que le maquillage toponymique dissimule maladroitement. Et quid des «Balkans », pour continuer notre inventaire? Dans certains cas, un même lieu peut être perçu comme étant situé en Europe centrale, en Europe médiane, en Mitteleuropa, en Europe orientale, dans les Balkans !

Ces toponymes sont révélateurs de la portée des stéréotypes mais aussi de l'extraordinaire complexité historique de certains lieux, de leur nature stratigraphique. Je n’irais pas jusqu'à dire dans un contexte aussi subtil que la géocritique est résolutoire, mais, pour le moins, me semble-t-il, elle peut apporter quelques outils et faciliter l'émergence de propositions. On notera au demeurant que ces définitions flottantes de grands « ensembles » constituent souvent une obsession européenne et indéniablement eurocentrique. Dans La cage des méridiens, j'ai beaucoup parlé de l' "Occident » et de l' "Orient », deux notions aussi schématiques voire fantaisistes l'une que l'autre, ce que Edward Said avait bien compris et montré. Il y a deux ans, je me suis retrouvé en difficulté, dans un texte sur le Liban, au moment d'avoir à traduire « Proche-Orient » et «Moyen-Orient » en anglais, langue dans laquelle n'existe couramment que le Middle East (le Near East est tombé en désuétude). En plus, en français et en anglais, quel que 
soit le terme que l'on sélectionne, il ne renverra pas aux mêmes espaces. Or, me suis-je dit, il se trouve que cet "Orient », qu'il soit proche, moyen ou middle, est avant tout occidental, mais pas au sens habituel - eurocentrique - de l'épithète. « Occidental », oui, mais en ce que le Liban se situe en « Asie occidentale».

Interviewer: La géocritique est devenue particulièrement pertinente pour l'étude de la littérature mimétique, liée aux espaces réels, même si la méthode que vous proposez n'exclut pas les genres littéraires ayant des géographies entièrement fictionnelles, comme la littérature fantastique ou la science-fiction. La géocritique serait-elle une méthodologie viable pour les études de poésie aussi ?

Bertrand Westphal: Effectivement, la géocritique avait initialement vocation à s'appliquer à des espaces dont le référent est ancré dans une géographie partagée, autrement dit en phase avec une cartographie établie par les géographes (tout en soulignant sa relativité !). Cependant, une fois encore, je crois qu'il convient de limiter la portée de ces premiers choix. On voudra bien admettre qu'en quinze ans j'ai repris quelques réflexions sous un angle différent ! S’il y a une et une seule raison pour laquelle je regretterais que mes derniers livres ne soient pas traduits en anglais c'est que les lecteurs anglophones n'auraient accès qu'à mes premiers titres. Or, à partir de La cage des méridiens et d'Atlas des égarements, un nouveau cycle se dessine dans ce que j'ai publié aux éditions de Minuit. Il prend une orientation légèrement différente, me semble-t-il, en tout cas complémentaire, sur le versant de la world literature et de la connexion entre littérature et art contemporain par l'intermédiaire de la cartographie qui est entendue ici au sens premier et non comme un vague "mapping ", une métaphore désormais très banale en anglais. Par le biais des arts visuels, on prend aisément conscience des multiples facettes du référent une fois qu'il est traduit sur le plan artistique et introduit dans une représentation qui se joue des modalités traditionnelles de la mimèsis.

Toutes mes premières études ont été consacrées à des lieux situés dans une géographique concrète. Cela dérivait aussi du fait que la réflexion théorique s'était appuyée au début sur l'examen d'un corpus à la fois ample et précis : le pourtour méditerranéen (voir L'œil de la Méditerranée, que j'ai cité tout à l'heure). En outre, se 
faisait sentir un impératif comparatiste, que j’ai depuis lors nettement modulé : celle de disposer de plusieurs appuis au niveau de la représentation par rapport à un référent précis, voire unique. Or une ville, une île, etc., relevant de la cartographie usuelle ont plus de chances d'être représentées au pluriel qu'un lieu imaginaire qui est propre à un auteur ou à une auteure plus qu'à une géographie partagée. Cela ne signifie en aucun cas que le cadre théorique soit figé. Il est sûr et certain que la science-fiction constitue un terrain d'études magnifique. Je parlais tout à l'heure de « laboratoire des possibles », or quel meilleur laboratoire y a-t-il que les univers science-fictionnels ou les mondes de l'heroic fantasy? Au demeurant, je crois que plusieurs études géocritiques sciencefictionnelles/fantasy ont été réalisées ces dernières années, ce dont je me réjouis. Il en va de même pour la poésie, qui d'ailleurs connaît plusieurs déclinaisons génériques. Faire la géocritique d'un sonnet, je ne sais pas, mais pourquoi pas... quant aux cycles poétiques plus longs, ils offrent de considérables possibilités. J'avoue être peu à l'aise dans ce domaine où des personnes infiniment plus qualifiées que moi pourraient intervenir. A vrai dire, un Kenneth White s'est beaucoup penché sur la poésie sous un angle géopoétique. La même remarque vaut pour l'écocritique. Bref, tout est envisageable dès lors qu'on a les compétences pour se lancer dans l'entreprise. Dans le même ordre d'idée, on m'a une fois reproché d'être trop « urbain » et pas assez attentif aux «grandes étendues» (nord-américaines). C'est encore une fois exact, mais que voulez-vous : nous ne sommes pas des machines et chacun réagit selon des réflexes culturels et - mais oui ! - selon ses goûts personnels... et voilà que refait surface la notion de subjectivité !

Interviewer: Après «le tournant spatial», les idées concernant l'espace et la géographie sont devenues essentielles pour les études culturelles et littéraires actuelle. Peut-on s'attendre à ce que la temporalité redevienne un paradigme dominant ? Ou, à l'avenir, le temps et l'espace seront-ils complètement reconsidérés dans le contexte scientifique global?

Bertrand Westphal: C'est difficile à dire. Ça l'est d'autant plus que la perception du temps et de l'espace dépassent infiniment nos facultés de perception immédiates. Il faut un recul considérable pour remarquer quoi que ce soit et émettre des hypothèses. Le 
Spatial Turn est intervenu au moment où le postmoderne et le postcolonial, se rencontrant, sont devenus dominants et ont arraché la culture, modélisée à partir de préceptes strictement occidentaux, à sa visée universaliste. Dans une vision classiciste et élitiste, seule la notion de temporalité était censée être en mesure de fournir les conditions d'un essor. Cet essor était lui-même perçu comme un progrès systématique, c'est-à-dire une amélioration du Même sur la base d'un discours épidictique qu'on s'adressait à soi-même. Je ne vais pas revenir sur ce que beaucoup de théoriciens et moimême avons pu développer depuis un bon demi-siècle pour certains (Henri Lefebvre, par exemple). Le Spatial Turn s'impose à partir de l'instant où l'on admet qu'il existe des modèles divers situés dans des endroits divers et, dans une perspective antithétique, que la compression spatio-temporelle les met tous en contact. On assiste alors à un double mouvement de lissage globalisant et d'hétérogénéisation culturelle, dont la synthèse est hautement problématique - la question étant de savoir si l'hétérogène est en mesure de résister et de survivre au processus de lissage (Edouard Glissant n'a cessé de réfléchir à ces question).

Sur ces prémisses, je me dis que le retour du temps ne serait pas nécessairement un signal encourageant, surtout si ce « temps » était censé participer d'une temporalité à nouveau uniforme : le Temps traditionnel, $\mathrm{t}$ majuscule. Certes, les choses ont beaucoup bougé depuis quelques décennies, au point qu'en 2016 Pheng Cheah a proposé de revoir, tout en nuance, le worlding culturel et littéraire en s'appuyant essentiellement sur des paramètres temporels dans What is a world ? que j'ai cité tout à l'heure. Cependant, je continue de me dire qu'il n'y a pas de duel ou de match entre le temps et l'espace, dont l'un ou l'autre sortirait vainqueur. J'ai toujours estimé qu'on ne pouvait parler du temps sans parler de l'espace, et inversement. Voilà pourquoi la temporalité et la spatialité ont toujours convergé dans une spatio-temporalité, dans mes travaux de géocritique, en 1995, en 2007 et, toujours encore, en 2020. Le Spatial Turn n'a au demeurant pas « chassé » la temporalité. Comment quelqu'un comme Fredric Jameson aurait-il pu vouloir cela ? Le Spatial Turn a plutôt permis un rééquilibrage. Si le paradigme spatial avait vraiment chassé le paradigme temporel, nous nous trouverions face à un sérieux problème. Inversement, si le temporel reprenait le dessus au détriment du spatial, j’ai l'impression - suivant le raisonnement à peine formulé - 
qu'il nous resterait à constater le triomphe du global. Ce n'est pas exactement la perspective que je trouve la plus exaltante! 